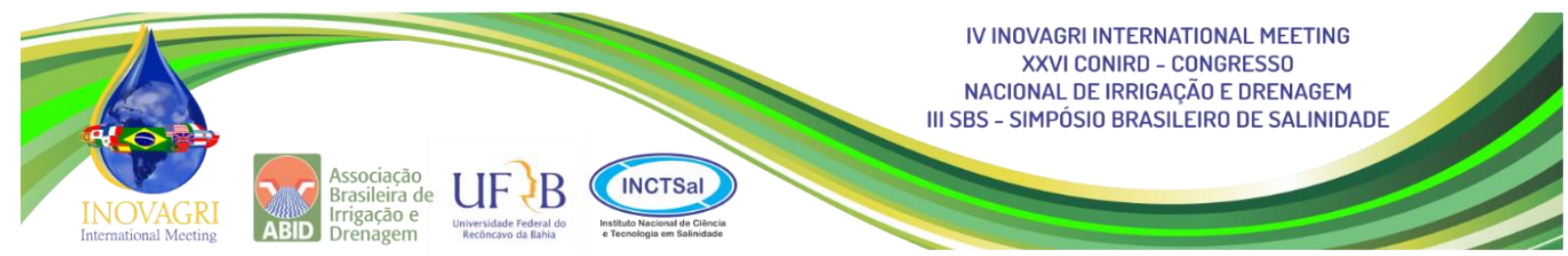

\title{
PRODUÇÃO DA VIDEIRA “ITALIA" SUBMETIDA A IRRIGAÇÃO COM DIFERENTES FRAÇÕES DA EVAPOTRANSPIRAÇÃO DA CULTURA
}

\author{
W. de B. Pereira ${ }^{1}$, J. S. C. de Sousa ${ }^{2}$, C. M. G. Santos ${ }^{3}$, W. L. Simões ${ }^{4}$, D. L. Oliveira ${ }^{3}$
}

RESUMO: O presente estudo foi realizado no Campus Petrolina Zona Rural do IF Sertão-PE, em Petrolina-PE, com o objetivo de estudar os fatores produtivos na produção da videira "Itália" quando submetida a irrigação com diferentes coeficientes de cultura no Vale do Submédio São Francisco. O delineamento experimental adotado foi o de blocos casualizados, com 6 tratamentos e 4 repetições, compreendendo, 24 unidades experimentais (a unidade experimental constituía-se de uma única planta). Os tratamentos foram nomeados de L1, L2, L3, L4, L5 e L6, referentes, respectivamente, a 25; 50; 75; 100; 125; 150\% do coeficiente de cultura (kc) sugerido por Soares \& Costa (2000). Observou-se que houve efeito significativo da lâmina para o número de ramos, assim como para número de cacho e produtividade média, e constatandose assim que o valor de kc que gerou os melhores resultados foi o de $100 \%$ da ETc.

PALAVRAS-CHAVE: Vitis vinífera, manejo de irrigação, gotejamento

\section{PRODUCTION OF "ITALIA" VINE SUBMITTED TO IRRIGATION WITH DIFFERENT FRACTIONS OF EVAPOTRANSPIRATION OF CULTURE}

\begin{abstract}
The present study was carried out at the Petrolina Campus Campus of the Sertão$\mathrm{PE}$, in Petrolina-PE, in order to study the production factors of the "Italia" grapevine when submitted to irrigation with different cultivation coefficients in the Vale do Submédio São Francisco. The experimental design was randomized blocks, with 6 treatments and 4 replicates, comprising 24 experimental units (the experimental unit consisted of a single plant). The treatments were named L1, L2, L3, L4, L5 and L6, respectively, at 25; 50; 75; 100; 125; 150\% of the crop coefficient (kc) suggested by Soares \& Costa (2000). It was observed that there was a significant effect of the lamina for the number of branches, as well as for bunch number and
\end{abstract}

\footnotetext{
${ }^{1}$ Discente de graduação em agronomia, IF-Sertão CPZR, Petrolina - Pernambuco. Email: wiltem1993if@gmail.com.

${ }^{2}$ Doutor, Discente de graduação em agronomia, IF-Sertão CPZR, Petrolina - Pernambuco. Email: sebastiao.costa@ifsertao-pe.edu.br.

${ }^{3}$ Doutor, Discente de graduação em agronomia, IF-Sertão CPZR, Petrolina - Pernambuco. Email: caiomaguisa@ hotmail.com.

${ }^{4}$ Doutor em Engenharia Agronômica, Pesquisador da Embrapa Semiárido, Petrolina - Pernambuco. Email: welson.simoes@embrapa.br.

${ }^{5}$ Discente de graduação em agronomia, IF-Sertão CPZR, Petrolina - Pernambuco. Email: daniloliveira10@hotmail.com.
} 
average productivity, and it was verified that the value of $\mathrm{kc}$ that generated the best results was $100 \%$ of ETc.

KEY WORDS: Vitis vinifera, irrigation management, drip irrigation

\section{INTRODUÇÃO}

Como afirmado por Silva \& Coelho (2010) o cultivo de uva de mesa é um dos mais expressivos no Submédio do Vale do São Francisco, em termos de quantidade de área cultivada, volume e qualidade das uvas produzidas.

Essa expressividade do cultivo da uva pode ser explicada pelo nível tecnológico que a região (Petrolina/Juazeiro) domina e dispõe e a rentabilidade do cultivo. Segundo Araújo (2010) a taxa de retorno médio da uva é da ordem de 1,88 (para cada $\mathrm{R} \$ 1,00$ investido obtém-se R\$ 1,88 de receita bruta).

O Vale do São Francisco possui uma característica ímpar na produção de uva, pelo fato de ser o único lugar no mundo capaz de produzir mais de duas safras por ano. Isso tem voltado os olhos de investidores para essa Região, fomentando a produção desse fruto nas localidades do médio São Francisco nas duas últimas décadas.

Para Souza et al. (2011) a cv. Itália é a principal representante das uvas finas de mesa (com sementes) explorada na região nordeste. Porém, os autores alertam que são verificados grandes variações de produtividades em razão dos manejos culturais adotados, uma vez que a cultura demanda de altos níveis tecnológicos (Correia \& Araújo, 2004).

Segundo Leão, (2010) a cv. Itália comum possui alta fertilidade a partir da quarta gema, adequando-se ao tipo de poda média (7 a 8 gemas vara-1). As folhas são de tamanho médio a grande com a parte inferior coberta por pelos. Os cachos são grandes com peso médio de $600 \mathrm{~g}$, cilíndrico-cônicos, alongados alados e muito compactos com boa resistência ao transporte e armazenagem. Possui bagas grandes de 8 a 12 g podendo chegar a mais de $23 \mathrm{~mm}$ de diâmetro, cor verde-amareladas e de consistência carnosa. De sabor levemente moscatel se tornando acentuado quando a colheita é realizada com o teor de sólidos solúveis totais acima de $16^{\circ}$ Brix. Possui produtividade na região semiárida de até $40 \mathrm{t} \mathrm{ha}^{-1}$ ano $^{-1}$ podendo chegar a $50 \mathrm{t} \mathrm{ha}^{-1}$ ano-

1. Muito sensível a doenças fúngicas sendo recomendado não fazer a poda em períodos de chuva.

Devido a esse fenômeno, muitas foram às pesquisas desenvolvidas por entidades como EMBRAPA (Empresa Brasileira de Pesquisa Agropecuária), IPA (Instituto Agronômico de Pernambuco), Universidades, Institutos Federais, etc., no intuito de definir adequadamente 
estratégias de manejo para a cultura da uva 'Itália' na região semiárida do Brasil. A citar os trabalhos como os apresentados por Soares \& Costa (2000) e Soares \& Leão (2009), adotados nos manejos de irrigação em praticamente todas as empresas do Perímetro Irrigado Senador Nilo Coelho (PISNC).

Contudo, ultimamente o Instituto Federal de Educação, Ciência e Tecnologia do Sertão Pernambucano (IF Sertão-PE), que sempre teve papel investigativo no que diz respeito às demandas regionais, vem recebendo questionamentos a respeito da real lâmina de irrigação para a uva 'Itália', oriundos, sobretudo de empresas de produção de uva e comerciantes de produtos agropecuários.

Desta forma, com este trabalho, buscou-se ajustar o manejo da irrigação para a uva 'Itália' na região do Vale do Submédio do São Francisco.

\section{MATERIAL E MÉTODOS}

O experimento foi realizado em um ciclo produtivo de uma área de produção de uva 'Itália' do Campus Petrolina Zona Rural do IF Sertão-PE, localizado nas coordenadas geográficas, $9^{\circ} 20^{\prime} \mathrm{Sul}, 40^{\circ} 41^{\prime}$ Oeste, altitude média de $418 \mathrm{~m}$.

O sistema de irrigação utilizado foi o gotejamento com duas fileiras de gotejadores por fileira de planta.

O delineamento experimental adotado foi o de blocos casualizados, com 6 tratamentos e 5 repetições, ou seja, 30 unidades experimentais (a unidade experimental constituía-se de uma única planta).

Os tratamentos foram:

L1 - 25\% do coeficiente de cultura (kc) sugerido por Soares \& Costa (2000).

L2 - 50\% do kc sugerido por Soares \& Costa (2000);

L3 - 75\% do kc sugerido por Soares \& Costa (2000);

L4 - 100\% do kc sugerido por Soares \& Costa (2000);

L5 - $125 \%$ do kc sugerido por Soares \& Costa (2000);

L6 - 150\% do Kc sugerido por Soares \& Costa (2000).

A irrigação da área era realizada de acordo com a ETo do dia anterior, sendo esse dado conseguido através da estação meteorológica do campus, o mesmo era inserido na planilha de irrigação onde gerava o tempo da irrigação para cada tratamento, sendo realizada diariamente, preferencialmente pela manhã. 
Os cálculos de manejo da irrigação foram realizados a partir da metodologia apresentada nos trabalhos de Vermeiren \& Jobling (1997), Gomes (1999), Bernardo el at., (2008), Mantovani et al., (2009) e Sousa (2012).

Foram instalados dois tensiômetros de punção (a 0,20; 0,40 e 0,60 m de profundidade) em três parcelas experimentais, constituíram 18 tensiômetros por profundidade, para monitoramento da umidade do solo. Está variável foram computados a partir de informações tensiométricas, aferidas três vezes por semana com uso de tensímetro digital.

As atividades deram-se início a partir do período da poda da videira e as variáveis avaliadas foram os números de ramos e de cacho por planta, produtividade média.

\section{Colheita}

A colheita foi realizada após 115 dias da poda (14/03/2016 a 07/07/2016).

Foram distribuídos contentores na área e realizada de forma separada por tratamento. No decorrer da colheita, foram separados quatro cachos de cada planta (unidade amostral), colocados em um saco plástico, identificados e levados ao laboratório, junto com toda produção, para as análises. No laboratório, os contentores foram pesados e armazenados na câmara fria. Já os sacos identificados, foram levados para a realização das análises de $\mathrm{pH}$, acidez e sólidos solúveis a partir dos métodos descritos por Morris (1948), Yemm \& Willis (1954), Miller (1959) e Pregnolatto \& Pregnolatto (1985).

Foi realizado a contabilização dos graus-dia acumulados para a colheita segundo metodologia proposta por Villa Nova et al. (1972), aplicando-se as equações 1 ou 2 e 3.

$$
\begin{array}{ll}
\mathrm{GDi}=\mathrm{T}-\mathrm{Tb} & (\text { para } \mathrm{Tm}>\mathrm{Tb} \text { e } \mathrm{TM}<\mathrm{TB}) \\
\mathrm{GDi}=(\mathrm{TM}-\mathrm{Tb})+\frac{T M-T m}{2}-\frac{(T M-T B)^{2}}{[2 \cdot(T M-T m)]}(\text { para } \mathrm{Tm}>\mathrm{Tb} \text { e } \mathrm{TM}>\mathrm{TB}) & \text { Eq. } 2 \\
\mathrm{GD}=\sum G D i & \text { Eq. } 3
\end{array}
$$

Onde:

GDi - graus-dia acumulados no dia i;

$\mathrm{T}$ - temperatura média do ar no dia i, ${ }^{\circ} \mathrm{C}$;

$\mathrm{TM}$ - temperatura máxima do ar para o dia i, ${ }^{\circ} \mathrm{C}$;

$\mathrm{Tm}$ - temperatura mínima do ar para o dia i, ${ }^{\circ} \mathrm{C}$;

$\mathrm{Tb}$ - temperatura basal inferior, ${ }^{\circ} \mathrm{C}$;

$\mathrm{TB}$ - temperatura basal superior para a cultura, ${ }^{\circ} \mathrm{C}$.

\section{Análise estatística dos dados}


Os dados foram submetidos à análise de variância e regressão a partir do programa computacional SISVAR (Ferreira et al., 2008).

\section{RESULTADOS E DISCUSSÃO}

\section{Número de ramos por planta}

Para a variável número de ramos por planta, foi observado sifnificância dos tratamentos (Tabela 1).

Tabela 1 . Análise de variância para o número de ramos por planta.

\begin{tabular}{ccccc}
\hline FV & GL & SQ & QM & F \\
\hline Blocos & 3 & 743.125000 & 247.708333 & $5.1100 .0124^{*}$ \\
Tratamentos & 5 & 936.708333 & 187.341667 & $3.8650 .0189 *$ \\
Erro Resíduo & 20 & 727.125000 & 48.475000 & \\
\hline
\end{tabular}

Total $23 \quad 2406.958333$

Obs.: FV - fonte de variação; GL - graus de liberdade; SQ - soma dos quadrados médios; QM - quadrado médio; F - teste $\mathrm{F}$; ** significativo ao nível de $1 \%$ de probabilidade $(\mathrm{p}<.01)$; * significativo ao nível de $5 \%$ de probabilidade $(.01=<\mathrm{p}<$ $.05)$; ns não significativo ( $\mathrm{p}>=.05)$.

Com isso, a regessão gerada foi polinomial de segundo grau (Figura 1) com quantidade máxima de ramos por planta (47,44 ramos por planta) para o fator de adequação do kc igual a 0,99 ou $99 \%$ da ETc. A quantidade de ramos obtido nessse trabalho foi superior ao observados por (BORGHEZAN et al., 2011) quando investigou o crescimento vegetativo de videira em Santa Catarina.

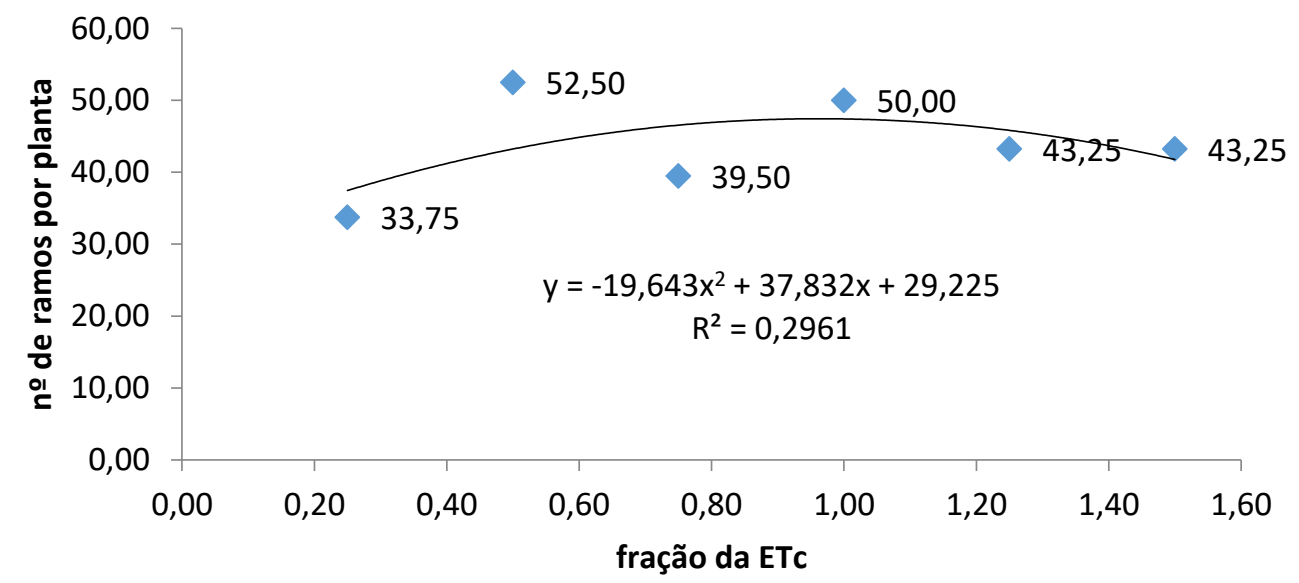

Figura 1. Regressão para número de ramos por planta. 


\section{Número de cachos por planta}

Para a variável números de cachos, observou-se não significância para os tratamentos conforme a Tabela 2. Contudo, foi realizado a análise de regressão que encontra-se ilustrado na Firura 2. Observa-se que o valor máximo de cachos por encontrado foi de 66,02 para o fator de adequação do kc igual a 0,94 ou 94\% da ETc. que difere com (GRANGEIRO et al, 2002) que teve uma produção de cachos de 19,30 por planta.

Tabela 2. Análise de variância para o número de cachos por planta.

\begin{tabular}{ccccc}
\hline FV & GL & SQ & QM & F \\
\hline Blocos & 3 & 660.166667 & 220.055556 & 0.8940 .4669 \\
Tratamentos & 5 & 3322.333333 & 664.466667 & 2.7000 .0621 \\
Resíduo & 15 & 3691.333333 & 246.088889 & \\
\hline Total & 23 & 7673.833333 & &
\end{tabular}

Obs.: FV - fonte de variação; GL - graus de liberdade; SQ - soma dos quadrados médios; QM - quadrado médio; F - teste F; ** significativo ao nível de $1 \%$ de probabilidade $(\mathrm{p}<.01)$; * significativo ao nível de $5 \%$ de probabilidade $(.01=<\mathrm{p}<.05)$; ns não significativo $(\mathrm{p}>=.05)$.

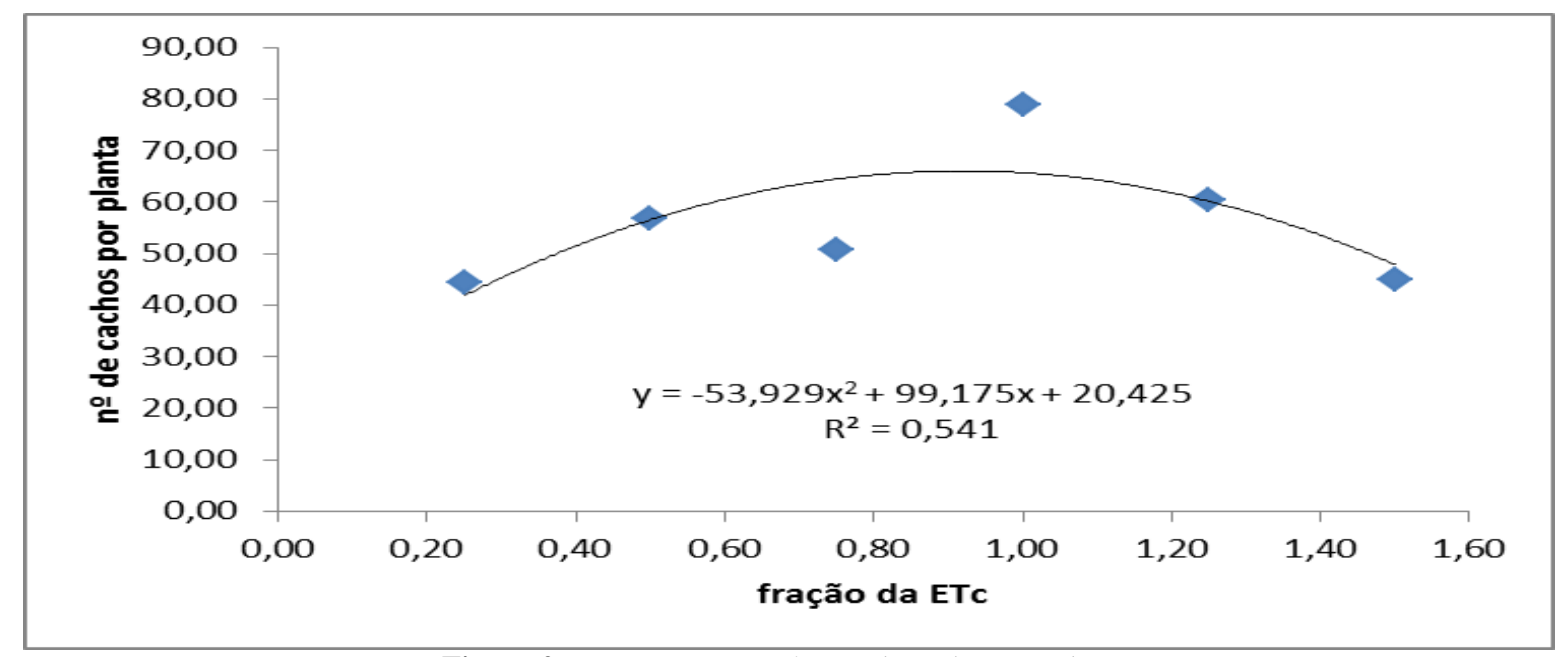

Figura 2. Regressão para número de cachos por planta.

\section{Produtividade média}

A produtividade média apresentou-se significância entre os tratamentos, de acordo com a Tabela 3. Com isso, realizou-se a análise de regressão que encontra-se ilustrado na Firura 3. Observou-se que a produtividade máxima obtida foi de 35,97 t ha-1 para um kc de $102 \%$ da ETc. Afirma (Albuquerque, 1996; Silva et al., 1998) que a produtividade é de 30 a 40 t/ha mencionadas para uva Itália no Vale do Rio São Francisco, diferente do trabalho realizado por (Grangeiro et al, 2002) que teve sua produtividade média de 5,30 $\mathrm{t} \mathrm{ha}^{-1}$. 
Tabela 3. Análise de variância para a produtividade média.

\begin{tabular}{ccccc}
\hline FV & GL & SQ & QM & F \\
\hline Blocos & 3 & 140.125000 & 46.708333 & 0.6970 .5682 \\
Tratamentos & 5 & 1231.208333 & 246.241667 & 3.6750 .0227 \\
Resíduo & 15 & 1005.125000 & 67.008333 & \\
\hline Total & 23 & 2376.458333 & &
\end{tabular}

Obs.: FV - fonte de variação; GL - graus de liberdade; SQ - soma dos quadrados médios; QM - quadrado médio; F - teste F; ** significativo ao nível de $1 \%$ de probabilidade $(\mathrm{p}<.01)$; * significativo ao nível de $5 \%$ de probabilidade $(.01=<\mathrm{p}<.05)$; ns não significativo $(\mathrm{p}>=.05)$.

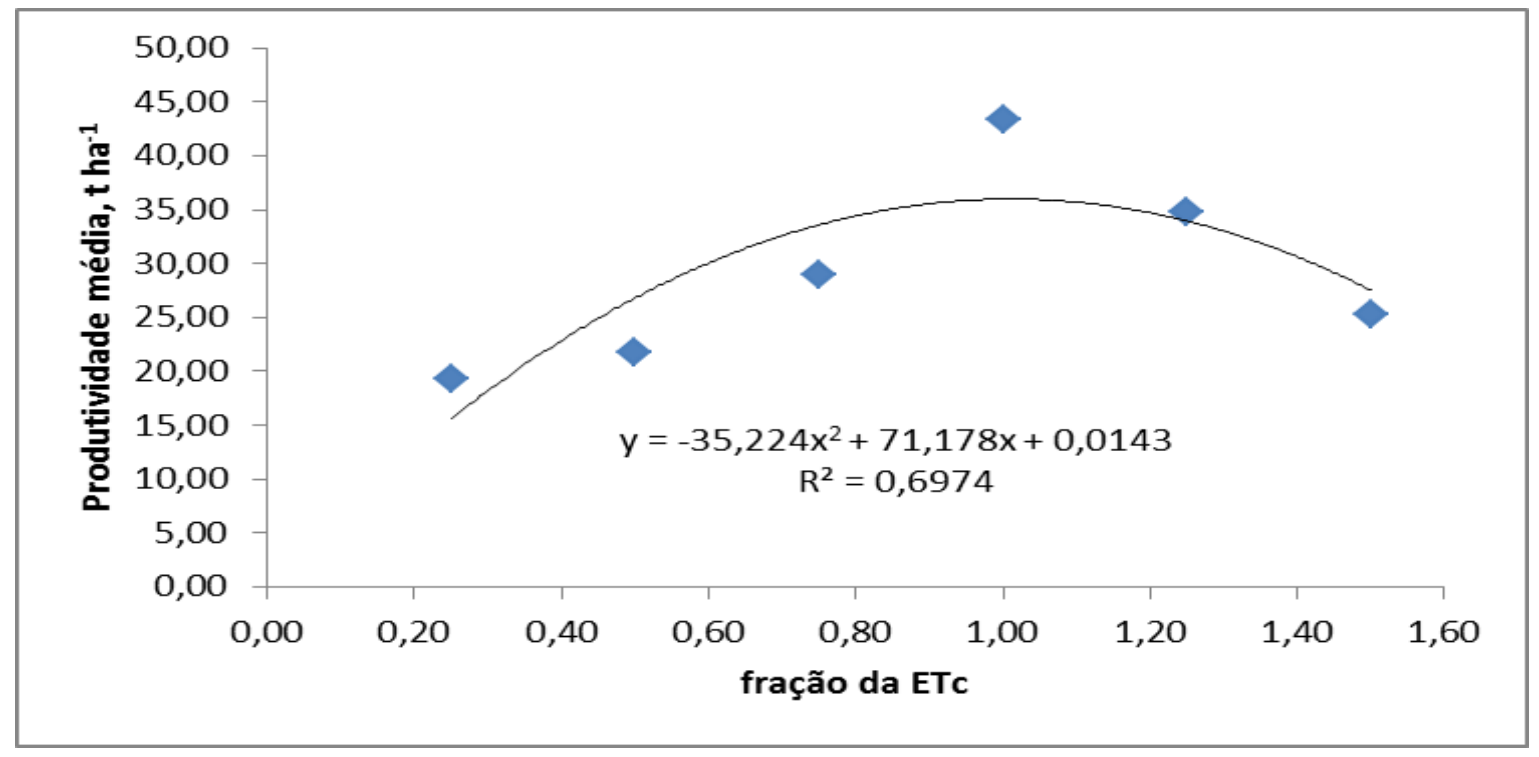

Figura 3. Regressão para produtividade média.

\section{Tensão de água no solo}

Para a variável tensão de água no solo foram registrados dados médios por profundidades de 0-0,20;0,20-0,40 e 0,40-0,60 m, para as diferentes fases fenológicas, apresentadas nas figuras 4 à 6. 
Tensões registradas na profundidade de $0-20 \mathrm{~cm}$

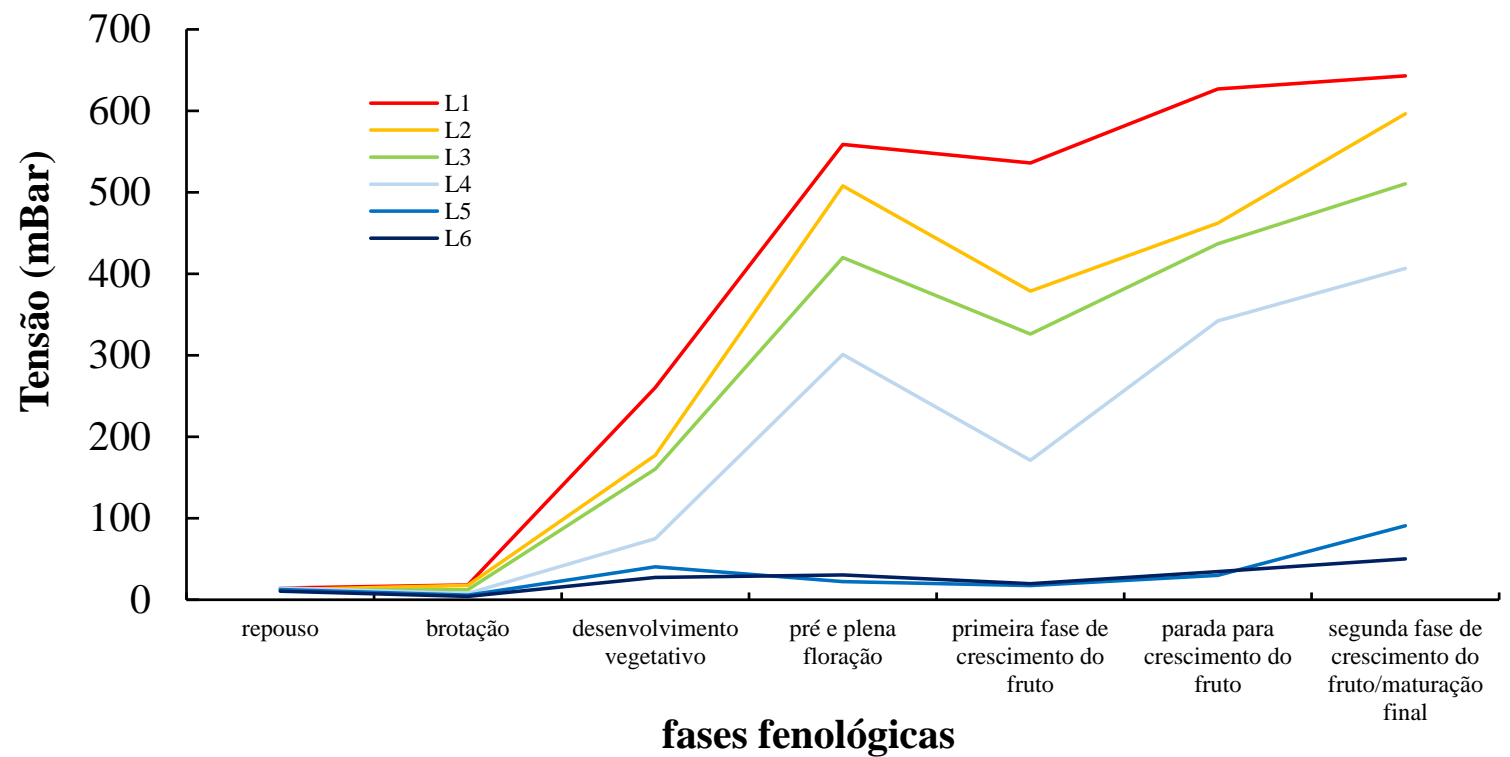

Figura 4. Potencial matricial do solo em na profundidade de 0-20 cm, em diferentes fases fenológicas.

Tensões registradas na profundidade de $20-40 \mathrm{~cm}$

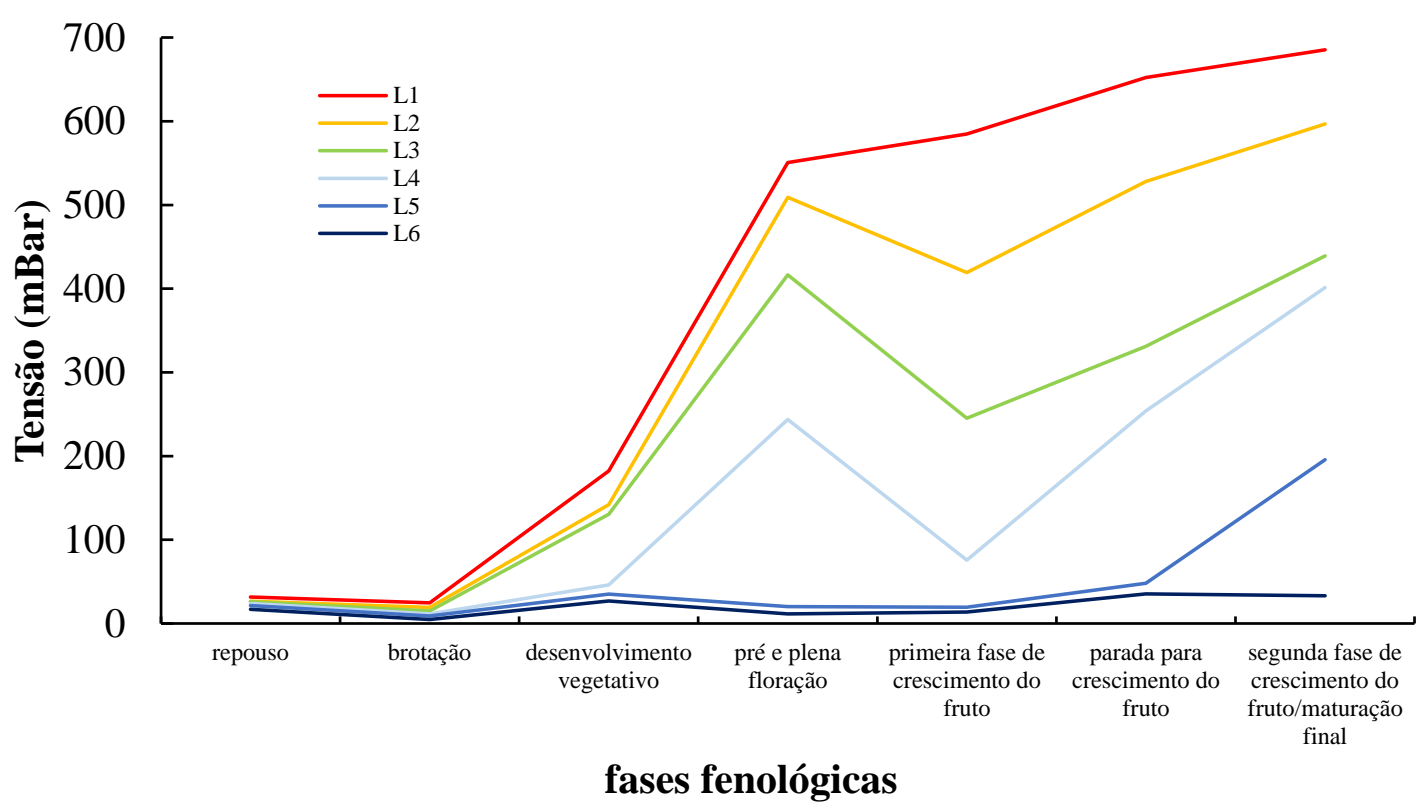

Figura 5. Potencial matricial do solo em na profundidade de $20-40 \mathrm{~cm}$, em diferentes fases fenológicas. 


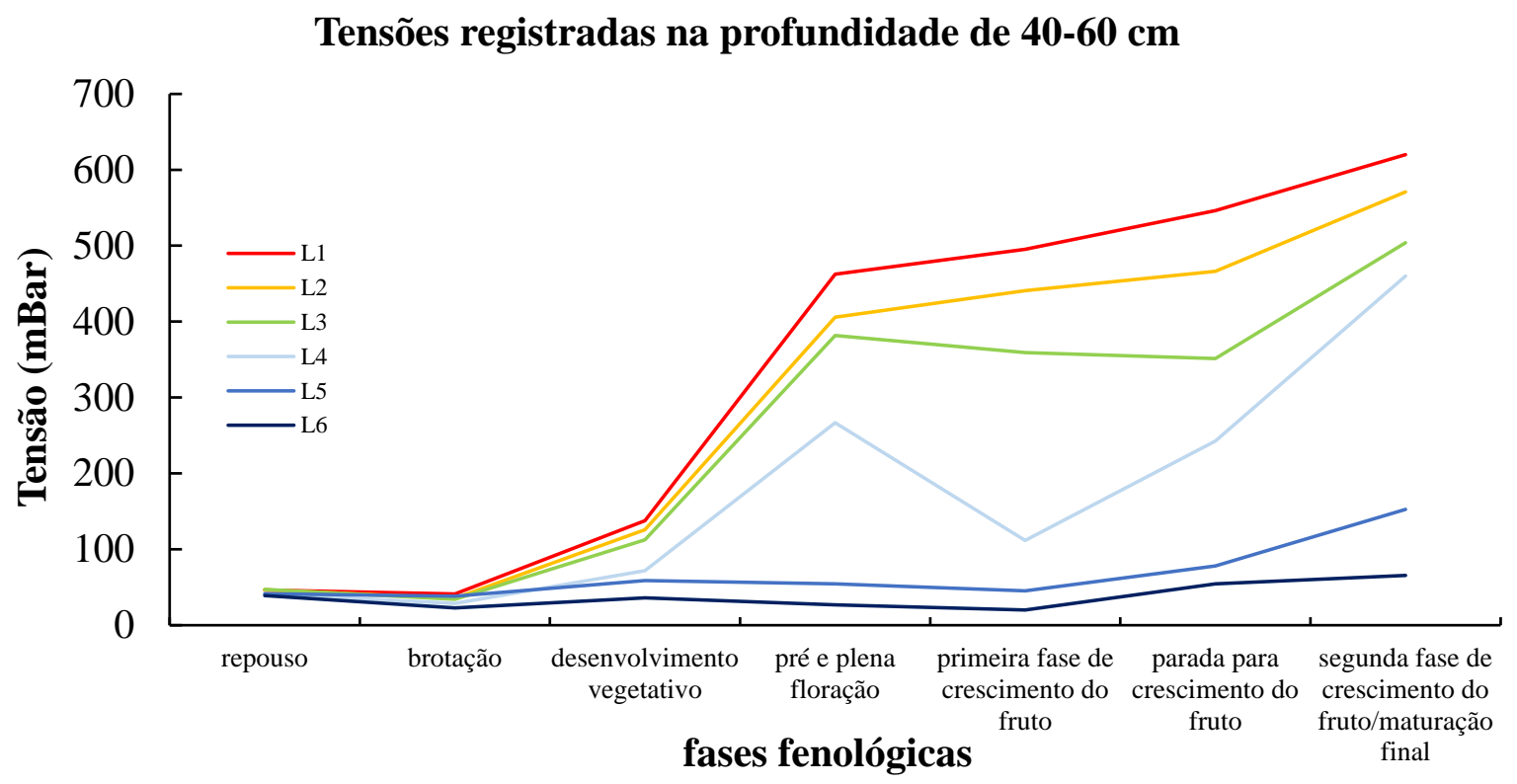

Figura 6. Potencial matricial do solo em na profundidade de 40-60 cm, em diferentes fases fenológicas.

\section{CONCLUSÕES}

Observou-se que a maior quantidade de ramos por planta $\left(47,44\right.$ ramos $\left._{\text {planta }}{ }^{-1}\right)$ foi conseguido com lâmina de irrigação igual a 99\% da ETc. Já a quantidade máxima de cacho por planta alcançada foi de 62,02 para lâmina de $94 \%$ da ETc, e a produtividade máxima foi de 35,97 t ha ${ }^{-1}$ para uma lâmina de $102 \%$ da ETc. Desta forma, lâminas de irrigação em torno de $100 \%$ da ETc foram as que geraram os melhores resultados.

\section{REFERÊNCIAS}

BORGHEZAN, M.; GAVIOLI, O.; PIT, F.A.; SILCA, A.L. da. Comportamento vegetativo e produtivo da videira e composição da uva em São Joaquim, Santa Catarina. Pesquisa Agropecuária Brasileira, Brasília, v.46, n.4, p.398-405, 2011.

CORREIA, R. C.; ARAUJO, J. L. P. Cultivo da Videira - Mercado Interno; Mercado externo; Características do mercado. Embrapa Semiárido: Sistemas de Produção, 1 ISSN 1807-0027. Versão Eletrônica Julho/2004. Disponível em: <https://ainfo.cnptia.embrapa.br/digital/bitstream/item/112196/1/Cultivo-da-videira $32070>$. Acesso em: 20 junho. 2017. 
GRANGEIRO, L. C.; LEÃO, P. C. DE S.; SOARES, J. M. Caracterização fenológica e produtiva da variedade de uva superior seedless cultivada no vale do são francisco. Rev. Bras. Frutic., Jaboticabal - SP, v. 24, n. 2, p. 552-554, agosto 2002.

MANTOVANI, E. C.; BERNARDO, S.; PALARETTI, L. F. Irrigação: princípios e métodos. 3 ed., atualizada e ampliada, Viçosa: Ed. UFV, 2009. 355p.

MILLER, G. L. Use of dinitrosalicylis acid reagent for determination of reducing sugars. Analytical Chemistry, Washington, DC, v. 31, n. 4, p. 426-428, 1959.

MORRIS, D. L. Quantitative determination of carbohydrates with drywood'santhrone reagent. Science, Washington, DC, v. 107, n. 1, p. 254-255, 1948.

PREGNOLATTO, W.; PREGNOLATTO, N. P. Normas analíticas do Instituto Adolfo Lutz: métodos químicos e físicos para análise de alimentos. 3. ed. São Paulo: Instituto Adolfo Lutz, v. $1,1985$.

SILVA, G. P. C.; COELHO, R. C. Caracterização social e econômica da cultura da videira. In: LEÃO, P. C. S. Cultivo da Videira. Embrapa Semiárido, Sistema de Produção 2 ed. Versão Eletrônica. 2010. Disponível em: <http://Sistemasdeproducao.cnptia.embrapa.br/> . Acesso em: 25 maio. 2016.

SILVA, P. C. G. da, LEÃO, P. C. de SOUZA; CERDAN, C., DENIS, S.; CHOUDHURY, M. M., BENTZEN, M. da C. P.; BARRETO, M. C. A cadeia produtiva da uva de mesa do Nordeste do Brasil. In: CASTRO, A. M. G. de; LIMA, S. M. V., GOEDERT, W. J., FILHO, A. de F.; VASCONCELOS, J. R. P. Cadeias produtivas e sistemas naturais: prospecção tecnológica. Brasília: EMBRAPA-SPI, 1998. p.529-64.

SOARES, J. M.; COSTA, F. F. Irrigação da cultura da videira. In: LEÃO, P. C. S., SOARES, J. M. A viticultura no semi-árido brasileiro. Petrolina: Embrapa Semi-árido, 2000. 368p.

SOARES, J. M.; LEÃO, P. C. S. A vitivinicultura no Semiárido brasileiro. Brasília, DF: Embrapa Informação Tecnológica; Petrolina: Embrapa Semiárido, versão atualizada, 2009. $756 \mathrm{p}$.

SOUZA, E. R.; RIBEIRO, V. G.; PIONÓRIO, J. A. A. Percentagem de fertilidade gemas e teores carboidratos contidos em raízes, sarmentos e folhas da videira cultivar Itália. Revista Brasileira de Tecnologia Aplicada nas Ciências Agrárias, Guarapuava-PR, v.4, n.1, p.8395, 2011. 
SOUSA, J. S. C. Adaptações de modelos matemáticos para dimensionamento hidráulico em sistemas de irrigação pressurizada. Campina Grande, UFCG, PB. 2012. 127p. (Tese de doutorado em Engenharia Agrícola).

VERMEIREN, G. A., JOBLING, G. A. Irrigação localizada. Campina Grande: UFPB, 1997, 184p. (Estudos FAO: Irrigação e Drenagem, 36 - Tradução de GHEYI, H. R., DAMASCENO, F. A. V., SILVA JUNIOR, L. G. A., MEDEIROS, J. F.).

VILLA NOVA, N. A. PEDRO JÚNIOR, M. J.; PERREIRA, A. R.; OMETTO, J. C. Estimativa de graus-dia acumulados acima de qualquer temperatura base em função das temperaturas máxima e mínima. Revista Ciência da Terra, São Paulo, n.30, p.1-8, 1972.

YEMM, E. W.; WILLIS, A. J. The estimation of carbohydrates in plants extracts by anthrone.

Biochemical Journal, Colchester, v. 57, n. 5, p. 508-514, 1954. 